\title{
Analysis of Finite Ground Plane Effects on Antenna Performance Using Discrete Green's Function
}

\author{
Salma Mirhadi, Mohammad Soleimani, and Ali Abdolali \\ Iran University of Science \& Technology, School of Electrical Engineering \\ Tehran, Iran \\ s_mirhadi@iust.ac.ir, soleimani@,iust.ac.ir, and $\underline{\text { Abdolali@iust.ac.ir }}$
}

\begin{abstract}
In this paper, the application of the discrete Green's function (DGF) method in the monopole antenna modeling with finite and infinite ground plane is presented. In the infinite ground plane case, the current distribution of antenna is obtained using image theory. While, in the finite ground plane case, the infinite free space DGF is used to obtain the current distribution on the antenna and ground plane. It is shown that the finite ground plane affects the properties of antenna, particularly the radiation pattern.
\end{abstract}

Keywords-component; current distribution, discrete Green's function; finite ground plane; monopole antenna.

\section{INTRODUCTION}

The initial concept of Discrete Green's Function (DGF) was developed by Vazquez and Parini in 1999 [1]. They derived the analytical closed form of the DGFs for infinite free space by the multidimensional z-transform of FDTD equations in time and spatial domains. They also showed that the field response of an arbitrary current source can be obtained as the convolution of the impulse response of the FDTD equations (DGF) and the current density. Then, they applied those expressions in antenna modeling by satisfying of the boundary conditions on the scatterers [2]. In fact, in this procedure, the induced currents on the antenna are updated instead of updating the fields in FDTD methods. Therefore, this formulation does not require absorbing boundary conditions or the computation of the free space nodes around the scatterers.

Other references, but not so much, presented further investigation into this area. In [3], Kastner described the need for an FDTD-compatible discrete Green's function instead of discretizing the continuous Green's functions directly. He also obtained another analytical expression for frequency and time domain of DGF. By modeling of a Yagi-Uda array antenna using DGF, Weili Ma et al. demonstrated considerable saving in computing time and memory storage compared with the traditional FDTD method [4]. Furthermore, Jeng derived new closed form expressions for the dyadic discrete Green's function in free space using the ordinary z-transform along with the spatial partial difference operators [5]. He claimed that the extracted expressions are easier than those in [1].

At this point, the DGFs have been fully derived and its property has been studied in detail. The next stage of work should be mainly concerned with more application of the DGF method, especially, in antenna modeling. Therefore, the aim of this paper is to apply DGF method to understand the effects of the finite ground plane on the antenna performance. Without loss of generality of this method, the monopole antenna has been studied. The current distribution along the monopole antenna as well as on the finite ground plane is determined using DGF method. We show that the radiation pattern for the monopole antenna is strongly affected by a finite sized ground plane while the impedance of a monopole antenna is minimally affected.

\section{DYADIC DISCRETE GREEN’S FUNCTION}

In this section, discrete Green's functions equations, derived in $[1-2,4]$, have been briefly mentioned. The Green's functions of the vector wave equation can be obtained through the Green's function of the scalar wave equation by applying the relationship between them. The discrete version of the scalar wave equation with Kronecker delta excitation can be expressed as:

$$
\begin{aligned}
& \frac{g_{i, j, k}^{n+1}-2 g_{i, j, k}^{n}+g_{i, j, k}^{n-1}}{c^{2}(\Delta t)^{2}}-\frac{g_{i+1, j, k}^{n}-2 g_{i, j, k}^{n}+g_{i-1, j, k}^{n}}{(\Delta x)^{2}} \\
& -\frac{g_{i, j+1, k}^{n}-2 g_{i, j, k}^{n}+g_{i, j-1, k}^{n}}{(\Delta y)^{2}}-\frac{g_{i, j, k+1}^{n}-2 g_{i, j, k}^{n}+g_{i, j, k-1}^{n}}{(\Delta z)^{2}}=\delta_{i-i^{\prime}, j-j^{\prime}, k-k^{\prime}}^{n-n^{\prime}}
\end{aligned}
$$

The solution of (1) can be achieved using the multidimensional $\mathrm{z}$ transform versus Jacobi polynomials as [1]:

$$
\begin{aligned}
& g_{i, j, k}^{n+1}=\sum_{m=0}^{n / 2}(-1)^{m}\left(\begin{array}{c}
n-m \\
m
\end{array}\right) \cdot \sum\left(n-2 m ; p_{x}, p_{y}, p_{z}\right) \\
& \prod_{\substack{t=i, j, k \\
s=x, y, z}} \alpha_{s}^{p_{s}} J_{p_{s}-l}^{(l, l)}\left(\xi_{s}\right)\left(R_{2 s}-R_{1 s}\right)^{p_{s}-l}
\end{aligned}
$$

where

$$
\begin{aligned}
& \alpha_{s}=\frac{c^{2} \Delta t^{2}}{\Delta s^{2}}\left(\frac{\Delta x^{2}+\Delta y^{2}+\Delta z^{2}}{\Delta s^{2}}\right), \beta_{\mathrm{s}}=\frac{\alpha_{s}-1}{\alpha_{s}}, R_{1,2 s}=\beta_{\mathrm{s}} \pm \sqrt{\beta_{\mathrm{s}}^{2}-1} \\
& \xi_{s}=\frac{R_{1 s}+R_{2 s}}{R_{1 s}-R_{2 s}},\left(n-2 m ; p_{x}, p_{y}, p_{z}\right)=\frac{(n-2 m) !}{p_{x} ! p_{y} ! p_{z} !}
\end{aligned}
$$




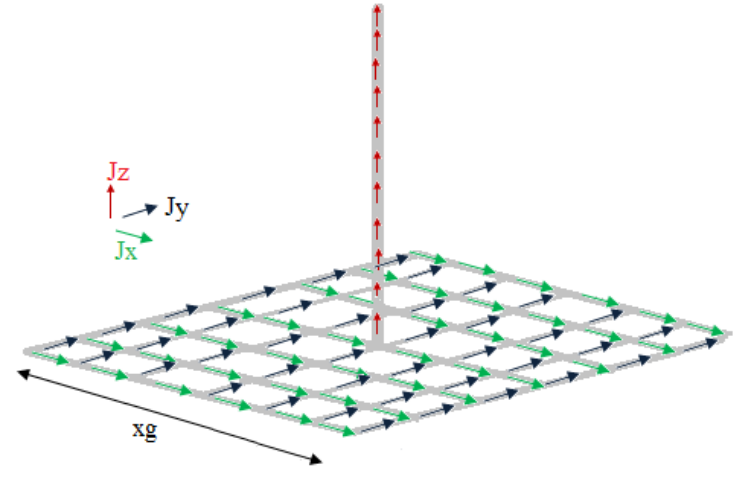

Figure 1. Current distribution of monopole antenna with finite ground plane in DGF analysis.

The vector Green's functions take the form of a matrix. For example, the vector Green's function relating the electric field to the electric current is as:

$$
\left[G_{e j}\right]_{i, j, k}^{n}=-\frac{\mu}{\Delta t}\left[\begin{array}{lll}
G_{x x} & G_{x y} & G_{x z} \\
G_{y x} & G_{y y} & G_{y z} \\
G_{z x} & G_{z y} & G_{z z}
\end{array}\right] g_{i, j, k}^{n}
$$

Where the matrix element are computed as:

$$
\begin{gathered}
{\left[G_{e j x x}\right]_{i, j, k}^{n}=g_{i, j, k}^{n}-g_{i, j, k}^{n-1}-\alpha_{x} \sum_{l=0}^{n} g_{i-1, j, k}^{l-1}-2 g_{i, j, k}^{l-1}+g_{i+1, j, k}^{l-1}} \\
{\left[G_{e j x y}\right]_{i, j, k}^{n}=\alpha_{x} \sum_{l=0}^{n} g_{i, j+1, k}^{l-1}-2 g_{i-1, j+1, k}^{l-1}+g_{i-1, j, k}^{l-1}-g_{i, j, k}^{l-1}} \\
{\left[G_{e j x z}\right]_{i, j, k}^{n}=\alpha_{x} \sum_{l=0}^{n} g_{i, j, k+1}^{l-1}-2 g_{i-1, j, k+1}^{l-1}+g_{i-1, j, k}^{l-1}-g_{i, j, k}^{l-1}}
\end{gathered}
$$

Other elements can be obtained similarly.

\section{MONOPOLE ANTENNA MODELING USING DGF}

In this section, modeling of monopole antenna with infinite and finite ground plane has been done using DGF. The mesh discretization and the current distribution of the monopole antenna and the finite ground plane are shown in Fig.1.

In the infinite ground plane case, the infinite free space DGF has been modified as (4) using image theory:

$$
\left[G_{-} \text {image }_{e j z z}\right]_{i, j, k, k^{\prime}}^{n}=\left[G_{e j z z}\right]_{i, j, k-k^{\prime}}^{n}+\left[G_{e j z z}\right]_{i, j, k+k^{\prime}}^{n}
$$

In this case, it is only necessary to compute the semiinfinite DGF along the antenna. Then, the update equation for the current on the antenna can be used as [2]:

$J_{z i, j, k}^{n}=-\frac{\varepsilon}{\Delta t}\left(E_{z i, j, k_{i n c}}^{n+1}-E_{z i, j, k i n c}^{n}\right)-\sum_{n^{\prime}=0}^{n-1} \sum_{k^{\prime}} J_{z i, j, k^{\prime}}^{n^{\prime}}\left[G_{-} \text {image }_{e j z z}\right]_{i, j, k, k^{\prime}}^{n}$

However, in the finite ground plane case, we use the infinite free space DGF and compute the all components of the

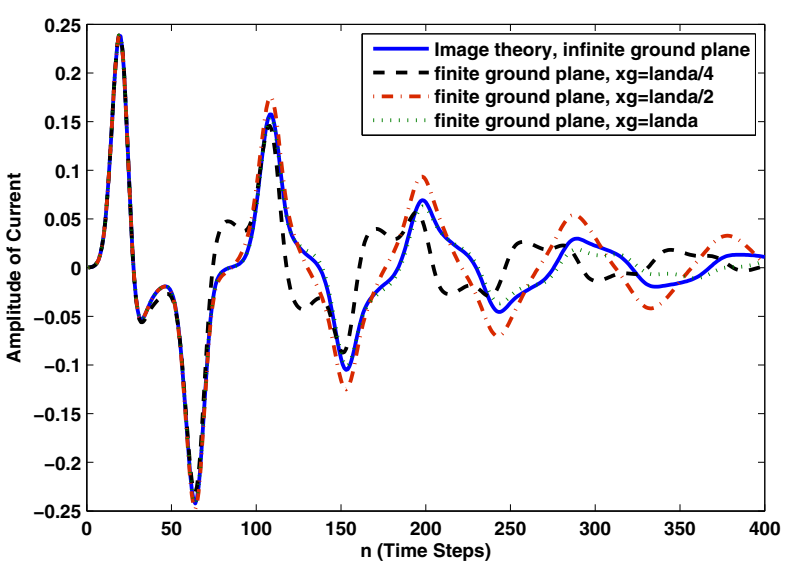

Figure 2. Temporal Response of Current at the feed point to the Gaussian incident field, $\mathrm{xg}$ ( the side length of the square ground plane).

dyadic DGF on the antenna and the finite ground plane. In other words, in the infinite ground plane case, the current on the monopole antenna in one dimension $\left(\mathrm{J}_{\mathrm{z}}\right)$ has been calculated. While, in the finite ground plane case, the one dimension current on the antenna and two dimension currents on the ground plane $\left(\mathrm{J}_{\mathrm{x}}\right.$ and $\left.\mathrm{J}_{\mathrm{y}}\right)$ have been calculated.

The monopole antenna with the length of $\lambda / 4$ at $300 \mathrm{MHz}$ has been studied. The spatial and time increments have been selected as $\Delta x=\Delta y=\Delta z=\lambda / 40, c \Delta t=0.5 \Delta x$ respectively. The antenna is excited by the incident Gaussian electric field. The temporal responses of current at the feed point are shown in Fig.2. As we can see, the smaller the ground plane, the sooner the current change compared to the infinite ground plane case due to the fact that the reflection of the ground plane edge occurs faster.

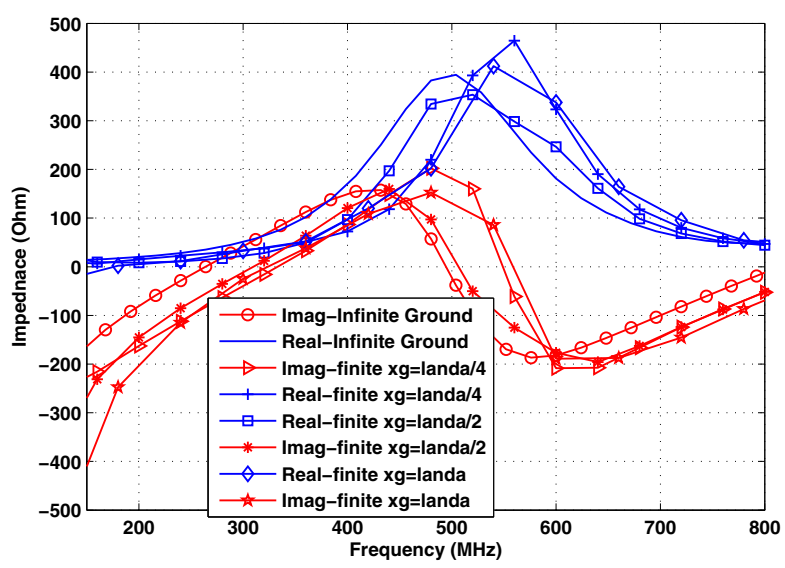

Figure 3. Imedance of monopole antenna with various groun plane size.

The real and imaginary part of impedance for different plane sizes is shown in Fig.3. The impedance curve for finite ground plane does not dramatically change compared to infinite ground plane case. It can be seen from the figure that, 
at $300 \mathrm{MHz}$, the imaginary part of impedance can be negative for the finite ground plane.

The far field radiation pattern of antenna can be determined from the magnetic vector potential obtained through the integration of current. As we can see in Fig. 4, the radiation pattern is strongly affected by the size of ground plane. The direction of peak-radiation has changed from the $\mathrm{x}$ $y$ plane to an angle elevated from that finite ground plane. There is also back radiation that reduced with increasing ground plane size.

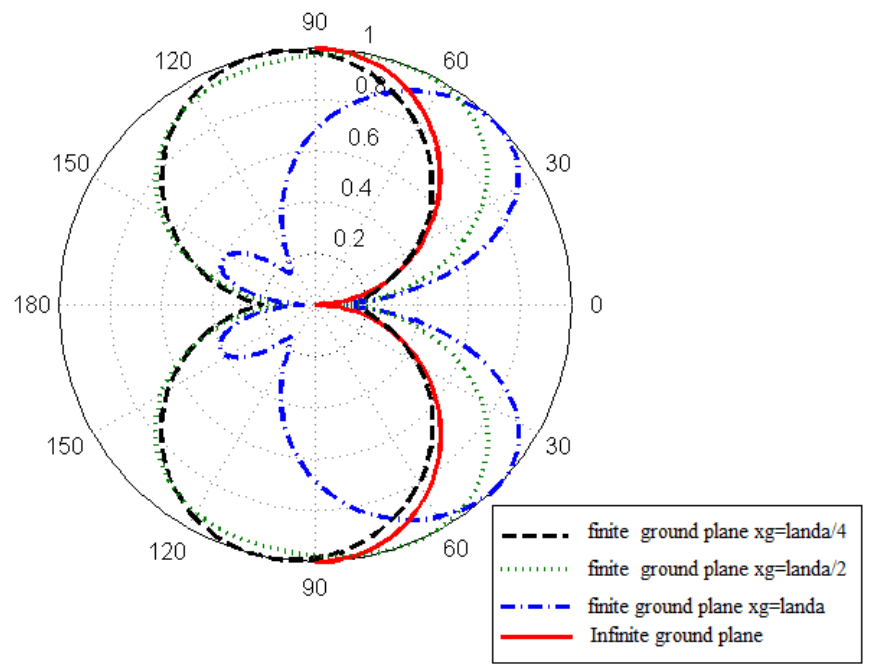

Figure 4. Radiation pattern of monopole antenna with various groun plane size.

\section{REFERENCES}

[1] J. Vazquez and C.G. Parini, "Discrete Green's function formulation of FDTD method for electromagnetic modelling," ELECTRONICS LETTER, vol. 35, No. 7, pp. 554-555, April 1999.

[2] J. Vazquez and C.G. Parini, "Anetnna modelling using discrete Green's function formulation of FDTD method," ELECTRONICS LETTER, vol. 35, No. 13, pp. 1033-1034, June 1999.

[3] R. Kastner, " A Multidimensional z-transform evaluation of the discrete finite difference time domian Green's function," IEEE Trans. Antennas Propag., vol. 54, No.4, pp. 1215-1222, April 2006.

[4] W. Ma, M. R. Rayner, and C. G. Parini, "Disctere Green's Function Formulation of the FDTD Method and Its Application in Antenna Modelin," IEEE Trans. Antennas Propag., vol. 53, No.1, pp. 339-364, January 2005.

[5] S. K. Jeng, "An Analytical Expresion for 3-D Dyadic FDTD-Compatible Green's Function in Infinite Free Space via z-Transform and Partial Difference Operators," IEEE Trans. Antennas Propag., vol. 59, No.4, pp. 1347-1355, April 2011. 